\title{
Existence and Multiplicity of Positive Solutions for Schrödinger-Kirchhoff-Poisson System with Singularity
}

\author{
Mengjun Mu and Huiqin Lu \\ School of Mathematical Sciences, Shandong Normal University, Jinan, Shandong 250014, China \\ Correspondence should be addressed to Huiqin Lu; lhy@sdu.edu.cn
}

Received 6 February 2017; Accepted 21 March 2017; Published 23 April 2017

Academic Editor: Guozhen Lu

Copyright (c) 2017 Mengjun Mu and Huiqin Lu. This is an open access article distributed under the Creative Commons Attribution License, which permits unrestricted use, distribution, and reproduction in any medium, provided the original work is properly cited.

We study a singular Schrödinger-Kirchhoff-Poisson system by the variational methods and the Nehari manifold and we prove the existence, uniqueness, and multiplicity of positive solutions of the problem under different conditions.

\section{Introduction}

In the present paper, we consider the following singular Schrödinger-Kirchhoff-Poisson system:

$$
\begin{aligned}
-\left(a+b \int_{\Omega}|\nabla u|^{2} d x\right) \Delta u+\varepsilon \eta \phi u & =\lambda f(x) u^{-\beta}, \quad \text { in } \Omega, \\
-\Delta \phi & =\eta u^{2}, \quad \text { in } \Omega, \\
u & >0, \quad \text { in } \Omega, \\
u & =\phi=0, \quad \text { on } \partial \Omega,
\end{aligned}
$$

where $\Omega \subset R^{3}$ is a smooth bounded domain with boundary $\partial \Omega, \quad a>0, b \geq 0, \eta \geq 0$, and $\lambda>0$ are four parameters and $\varepsilon= \pm 1, \beta \in(0,1)$ is a constant, and $f(x) \in C^{1}(\bar{\Omega})$ is a nontrivial nonnegative function.

When $a=1, b=0, \eta=1$, and $f(x) \equiv 1$, system (1) reduces to the following singular Schrödinger-Poisson system:

$$
\begin{aligned}
-\Delta u+\varepsilon \phi u & =\mu u^{-\beta}, \quad \text { in } \Omega, \\
-\Delta \phi & =u^{2}, \quad \text { in } \Omega, \\
u & >0, \quad \text { in } \Omega, \\
u & =\phi=0, \quad \text { on } \partial \Omega,
\end{aligned}
$$

which has been studied in [1]. By using the variational method and the Nehari manifold, the existence, uniqueness, and multiplicity of solutions for system (2) have been obtained.

When $a=1, b=0$, and $\eta=0$, system (1) reduces to the following singular semilinear elliptic problem:

$$
\begin{aligned}
\Delta u+\lambda f(x) u^{-\beta} & =0, & \text { in } \Omega, \\
u & =0, & \text { on } \partial \Omega ;
\end{aligned}
$$

the existence and uniqueness of positive solution have been studied in $[2,3]$ for $\lambda=1$.

When $\eta=0$, the system (1) reduces to the following singular Kirchhoff type problem:

$$
\begin{aligned}
-\left(a+b \int_{\Omega}|\nabla u|^{2} d x\right) \Delta u & =\lambda f(x) u^{-\beta}, \quad \text { in } \Omega, \\
u & =0, \quad \text { on } \partial \Omega .
\end{aligned}
$$

It is well known that the following Kichhoff type problem:

$$
\begin{aligned}
-\left(a+b \int_{\Omega}|\nabla u|^{2} d x\right) \Delta u & =h(x, u), \quad \text { in } \Omega, \\
u & =0, \quad \text { on } \partial \Omega,
\end{aligned}
$$

where $\Omega \subset R^{3}$ is a smooth bounded domain and $h: \bar{\Omega} \times$ $R \rightarrow R$ is a continuous function, has been extensively studied; 
see [4-7] and so forth. Problem (5) is called nonlocal because of the presence of the term $\int_{\Omega}|\nabla u|^{2} d x$ which implies, when $b \neq 0$, that the equation in (5) is no longer a pointwise identity.

Recently, the singular Kirchhoff type problems have been considered (see [8-11]). In [8], by using the Nehari manifold methods, Liu and Sun proved that problem (5) with $h(x)=$ $f(x) u^{-\beta}+\lambda g(x)\left(u^{p} /|x|^{s}\right)$ has at least two positive solutions for $\lambda>0$ small enough. In [9], by using the variational methods, Lei et al. obtained that problem (5) with $h(x)=u^{5}+\mu u^{-\beta}$ has at least two positive solutions for $\mu>0$ small enough. The common characteristic of [8-10] is that the nonlinear terms contain both singular and superlinear. The superlinear term $u^{p}(p \geq 3)$ can overcome the difficulties which are caused by the Kirchhoff type perturbation $\|u\|_{H_{0}^{1}(\Omega)}^{4}$.

Motivated by the above references, especially by [8-10], we study the singular Schrödinger-Kirchhoff-Poisson system (1). Let $H_{0}^{1}(\Omega)$ be the Sobolev space equipped with the inner product and norm

$$
(u, v)=\int_{\Omega} \nabla u \cdot \nabla v, \quad\|u\|=(u, u)^{1 / 2} .
$$

As usual, $|u|_{s}=\left(\int_{\Omega}|\nabla u|^{s}\right)^{1 / s}, s \in[1,+\infty)$, denotes the norm of the Lebesgue space $L^{s}(\Omega)$. Let $S>0$ be the usual Sobolev constant defined by

$$
\inf _{u \in H_{0}^{1}(\Omega) \backslash\{0\}} \frac{\int_{\Omega}|\nabla u|^{2} d x}{\left(\int_{\Omega} u^{6} d x\right)^{1 / 3}} ;
$$

that is,

$$
|u|_{6}^{2} \leq S^{-1}\|u\|^{2}, \quad \forall u \in H_{0}^{1}(\Omega) .
$$

By the Hölder inequality and (8), we have

$$
\begin{aligned}
\int_{\Omega} f(x)|u|^{1-\beta} & \leq|f|_{\infty}|u|_{4}^{1-\beta}|\Omega|^{(3+\beta) / 4} \\
& \leq|f|_{\infty}|u|_{6}^{1-\beta}|\Omega|^{(5+\beta) / 6} \\
& \leq|f|_{\infty} S^{-(1-\beta) / 2}\|u\|^{1-\beta}|\Omega|^{(5+\beta) / 6}
\end{aligned}
$$

where $|\Omega|$ denotes the Lebesgue measure of the domain $\Omega$.

Before we state the main results about system (1), we first recall the following well-known facts (see [1]).

Lemma 1. Let $\eta \geq 0$; then for every $u \in H_{0}^{1}(\Omega)$, there exists a unique $\phi_{u} \in H_{0}^{1}(\Omega)$ solution of

$$
\begin{aligned}
-\Delta \phi & =\eta u^{2}, \quad \text { in } \Omega, \\
u & =0, \quad \text { on } \partial \Omega .
\end{aligned}
$$

Moreover

(i) $\left\|\phi_{u}\right\|^{2}=\eta \int_{\Omega} \phi_{u}|u|^{2}$;

(ii) $\phi_{u} \geq 0$. Moreover $\phi_{u}>0$ when $u \neq 0$ and $\eta>0$;

(iii) for each $t \neq 0, \phi_{t u}=t^{2} \phi_{u}$; (iv)

$$
\begin{aligned}
\int_{\Omega} \phi_{u} u^{2} & \leq \eta S^{-1}|u|_{12 / 5}^{4} \leq \eta S^{-1}|u|_{4}^{4}|\Omega|^{2 / 3} \\
& \leq \eta S^{-3}\|u\|^{4}|\Omega|, \quad u \in H_{0}^{1}(\Omega) ;
\end{aligned}
$$

(v) assume that $u_{n} \rightarrow u$; then $\phi_{u_{n}} \rightarrow \phi_{u}$ in $H_{0}^{1}(\Omega)$ and $\int_{\Omega} \phi_{u_{n}} u_{n} v \rightarrow \int_{\Omega} \phi_{u} u v$ for any $v \in H_{0}^{1}(\Omega)$

(vi) $\phi_{u} \in W_{\text {loc }}^{2,3}(\Omega) \cap C^{0}(\bar{\Omega})$;

(vii) for $u, v \in H_{0}^{1}(\Omega), \eta \int_{\Omega}\left(\phi_{u} u-\phi_{v} v\right)(u-v) \geq(1 / 2) \| \phi_{u}-$ $\phi_{v} \|^{2}$.

By Lemma 1, we easily see that system (1) can be converted into a binonlocal type problem of the singular Schrödingerkirchhoff

$$
-\left(a+b \int_{\Omega}|\nabla u|^{2} d x\right) \Delta u+\varepsilon \eta \phi_{u} u=\lambda f(x) u^{-\beta},
$$

in $\Omega$,

$$
\begin{array}{ll}
u>0, & \text { in } \Omega, \\
u=0, & \text { on } \partial \Omega .
\end{array}
$$

We define the functional

$$
\begin{aligned}
J_{\lambda}(u)= & \frac{a}{2}\|u\|^{2}+\frac{b}{4}\|u\|^{4}+\frac{\varepsilon \eta}{4} \int_{\Omega} \phi_{u} u^{2} \\
& -\frac{\lambda}{1-\beta} \int_{\Omega} f(x)|u|^{1-\beta}, \quad u \in H_{0}^{1}(\Omega) .
\end{aligned}
$$

For any $u, v \in H_{0}^{1}(\Omega)$, by (9),

$$
\begin{aligned}
& \left|\int_{\Omega} f(x)\left(|u|^{1-\beta}-|v|^{1-\beta}\right)\right| \leq|f|_{\infty} \int_{\Omega}|u-v|^{1-\beta} \\
& \quad \leq|f|_{\infty}|u-v|_{4}^{1-\beta}|\Omega|^{(3+\beta) / 4} \\
& \quad \leq|f|_{\infty} S^{-(1-\beta) / 2}\|u-v\|^{1-\beta}|\Omega|^{(5+\beta) / 6}
\end{aligned}
$$

Then, by Lemma $1, J_{\lambda}(u)$ is well defined and continuous on $H_{0}^{1}(\Omega)$.

In general, a function $u$ is called a solution of (12), if $u \in$ $H_{0}^{1}(\Omega), u(x)>0, \forall x \in \Omega$, and

$$
\begin{gathered}
\left(a+b \int_{\Omega}|\nabla u|^{2}\right) \int_{\Omega} \nabla u \cdot \nabla v+\varepsilon \eta \int_{\Omega} \phi_{u} u v-\lambda \\
\cdot \int_{\Omega} f(x) u^{-\beta} v=0, \quad \forall v \in H_{0}^{1}(\Omega) .
\end{gathered}
$$

Moreover, $\left(u, \phi_{u}\right) \in H_{0}^{1}(\Omega) \times H_{0}^{1}(\Omega)$ is a solution of system (1) if and only if $u \in H_{0}^{1}(\Omega)$ is a solution of (12).

As far as we know, the singular Schrödinger-KirchhoffPoisson system has not been considered up to now, and the study on the existence, uniqueness, and multiplicity of solutions for system (1) is meaningful in mathematics. We emphasize that the combined effects of the two nonlocal 
terms it contains cause some mathematical difficulties which make the study of such class of problems particularly interesting.

We consider system (1) in two cases: $\varepsilon=1$ and $\varepsilon=-1$. By using the variational method, the Nehari manifold, and Ekeland's variational principle, we obtain the existence and uniqueness of positive solution for $\varepsilon=1$ and the multiplicity of positive solutions for $\varepsilon=-1$. To prove the multiplicity of positive solutions, it is necessary that $N_{\lambda}^{ \pm} \neq \emptyset$, where $N_{\lambda}^{ \pm}$are defined in Section 3.2. But the method of getting $N_{\lambda}^{ \pm} \neq \emptyset$ in [1] cannot apply to our problem. In order to show that $N_{\lambda}^{ \pm} \neq \emptyset$, we introduce a special nonempty set. Let

$$
\begin{aligned}
& \eta_{0}=b^{1 / 2} S^{3 / 2}|\Omega|^{-1 / 2}, \\
& \lambda_{*} \\
& =\frac{2 a}{3+\beta}\left[\frac{a(1+\beta)}{3+\beta}\right]^{(1+\beta) / 2} \frac{|f|_{\infty}^{-1} S^{(1-\beta) / 2}|\Omega|^{-(5+\beta) / 6}}{\left(\eta^{2} S^{-3}|\Omega|-b\right)^{(1+\beta) / 2}} .
\end{aligned}
$$

Our main results can be described as follows.

Theorem 2. Assume $a>0, b \geq 0, \varepsilon=1$, and $\beta \in(0,1)$, then system (1) has a unique positive solution for all $\eta \geq 0$ and $\lambda>0$.

Theorem 3. Assume $a>0, b \geq 0, \varepsilon=-1$, and $\beta \in(0,1)$, then

(i) When $0 \leq \eta \leq \eta_{0}$, system (1) has at least one positive solution for all $\lambda>0$;

(ii) When $\eta>\eta_{0}$, system (1) has at least two positive solutions for all $0<\lambda<\lambda_{*}$.

Remark 4. When $a=1, b=0, \eta=1$, and $f(x) \equiv 1$, we easily see that $\lambda_{*}=\mu^{*}$, where $\mu^{*}$ is as in Theorem 1.2 in [1]. Consequently, our results generalize and improve those of [1].

\section{Proof of Theorem 2}

Lemma 5. For all $a, \lambda>0, b, \eta \geq 0$, and $\varepsilon=1$, the functional $J_{\lambda}$ attains the global minimizer in $H_{0}^{1}(\Omega)$; that is, there exists $u_{*} \in H_{0}^{1}(\Omega)$ such that

$$
J_{\lambda}\left(u_{*}\right)=m_{\lambda}=\inf _{u \in H_{0}^{1}(\Omega)} J_{\lambda}(u)<0 .
$$

Proof of Lemma 5. For $u \in H_{0}^{1}(\Omega)$, by Lemma 1(ii) and (9),

$$
\begin{aligned}
J_{\lambda}(u) \geq & \frac{a}{2}\|u\|^{2} \\
& -\frac{\lambda}{1-\beta} S^{-(1-\beta) / 2}|f|_{\infty}|\Omega|^{(5+\beta) / 6}\|u\|^{1-\beta}
\end{aligned}
$$

so, $J_{\lambda}(u)$ is coercive and bounded from below on $H_{0}^{1}(\Omega)$ for any $\lambda>0$. Thus $m_{\lambda}=\inf _{u \in H_{0}^{1}(\Omega)} J_{u}(u)$ is well defined. For $t>0$ and given $u \in H_{0}^{1}(\Omega) \backslash\{0\}$,

$$
\begin{aligned}
J_{\lambda}(t u)= & \frac{a t^{2}}{2}\|u\|^{2}+\frac{b}{4} t^{4}\|u\|^{4}+\frac{\eta t^{4}}{4} \int_{\Omega} \phi_{u} u^{2} \\
& -\frac{\lambda}{1-\beta} t^{1-\beta} \int_{\Omega} f(x)|u|^{1-\beta} ;
\end{aligned}
$$

we can see that for $t>0$ small enough, $J_{\lambda}(t u)<0$. Therefore, we have $m_{\lambda}=\inf _{u \in H_{0}^{1}(\Omega)} J_{u}(u)<0$.

According to the definition of $m_{\lambda}$, there exists a minimizing sequence $\left\{u_{n}\right\} \subset H$ such that $\lim _{n \rightarrow \infty} J_{\lambda}\left(u_{n}\right)=m_{\lambda}<0$. Since $J_{\lambda}\left(u_{n}\right)=J_{\lambda}\left(\left|u_{n}\right|\right)$, we may assume that $u_{n} \geq 0$. From (18), it is easy to see that $\left\{u_{n}\right\}$ is bounded in $H_{0}^{1}(\Omega)$, up to a subsequence; there exists $u_{*} \in H_{0}^{1}(\Omega)$ such that

$$
\begin{gathered}
u_{n} \rightarrow u_{*}, \quad \text { in } H_{0}^{1}(\Omega) \\
u_{n} \longrightarrow u_{*}, \quad \text { in } L^{p}(\Omega), p \in[1,6) \\
u_{n}(x) \longrightarrow u_{*}(x), \quad \text { a.e. in } \Omega .
\end{gathered}
$$

Then by the weakly lower semicontinuity of the norm, Lemma $1(\mathrm{v})$ and (14), we have

$$
\begin{aligned}
m_{\lambda} \leq & J_{\lambda}\left(u_{*}\right) \\
= & \frac{a}{2}\left\|u_{*}\right\|^{2}+\frac{b}{4}\left\|u_{*}\right\|^{4}+\frac{\eta}{4} \int_{\Omega} \phi_{u_{*}} u_{*}^{2} \\
& -\frac{\lambda}{1-\beta} \int_{\Omega} f(x)\left|u_{*}\right|^{1-\beta} \leq \liminf _{n \rightarrow \infty} J_{\lambda}\left(u_{n}\right) \\
= & m_{\lambda} .
\end{aligned}
$$

So, we have $J_{\lambda}\left(u_{*}\right)=m_{\lambda}<0$.

Proof of Theorem 2. We divide three steps to prove Theorem 2.

(1) We show $u_{*}>0$ in $\Omega$.

From Lemma 5, $u_{*} \geq 0$ and $u_{*} \neq 0$. Fix $\varphi \in H_{0}^{1}(\Omega), \varphi>0$, and $t \geq 0$; we have

$$
\begin{aligned}
0 \leq & \lim _{t \rightarrow 0} \inf \frac{J_{\lambda}\left(u_{*}+t \varphi\right)-J_{\lambda}\left(u_{*}\right)}{t} \\
= & \int_{\Omega}\left[\left(a+b\left\|u_{*}\right\|^{2}\right) \nabla u_{*} \nabla \varphi+\eta \phi_{u_{*}} u_{*} \varphi\right] \\
& -\frac{\lambda}{1-\beta} \lim \operatorname{sub}_{t \rightarrow 0} \int_{\Omega} f(x) \frac{\left(u_{*}+t \varphi\right)^{1-\beta}-u_{*}^{1-\beta}}{t} ;
\end{aligned}
$$

that is

$$
\begin{aligned}
& \frac{\lambda}{1-\beta} \lim \operatorname{sub}_{t \rightarrow 0} \int_{\Omega} f(x) \frac{\left(u_{*}+t \varphi\right)^{1-\beta}-u_{*}^{1-\beta}}{t} \\
& \quad \leq \int_{\Omega}\left[\left(a+b\left\|u_{*}\right\|^{2}\right) \nabla u_{*} \nabla \varphi+\eta \phi_{u_{*}} u_{*} \varphi\right] .
\end{aligned}
$$


Notice that

$$
\begin{aligned}
& \int_{\Omega} f(x) \frac{\left(u_{*}+t \varphi\right)^{1-\beta}-u_{*}^{1-\beta}}{t} \\
& \quad=(1-\beta) \int_{\Omega} f(x)\left(u_{*}+t \varphi \xi\right)^{-\beta} \varphi,
\end{aligned}
$$

where $\xi(x) \in(0,1)$ and $\left(u_{*}(x)+t \varphi(x) \xi(x)\right)^{-\beta} \varphi(x) \rightarrow$ $\left(u_{*}(x)\right)^{-\beta} \varphi(x)$; a.e $x \in \Omega, t \rightarrow 0$. Since $\left(u_{*}(x)+\right.$ $t \varphi(x) \xi(x))^{-\beta} \varphi(x) \geq 0$, by using Fatou's lemma, we have

$$
\begin{aligned}
& \lambda \int_{\Omega} f(x) u_{*}^{-\beta} \varphi \\
& \quad \leq \int_{\Omega}\left[\left(a+b\left\|u_{*}\right\|^{2}\right) \nabla u_{*} \nabla \varphi+\eta \phi_{u_{*}} u_{*} \varphi\right] .
\end{aligned}
$$

By the idea of approximation, the above expression also holds for $\varphi \in H_{0}^{1}(\Omega), \varphi \geq 0$; that is,

$$
\begin{gathered}
\int_{\Omega}\left[\left(a+b\left\|u_{*}\right\|^{2}\right) \nabla u_{*} \nabla \varphi+\eta \phi_{u_{*}} u_{*} \varphi\right] \\
-\lambda \int_{\Omega} f(x) u_{*}^{-\beta} \varphi \geq 0 .
\end{gathered}
$$

Therefore,

$$
-\left(a+b\left\|u_{*}\right\|^{2}\right) \Delta u_{*}+\eta \phi_{u_{*}} u_{*} \geq 0
$$

in the weak sense.

Since $u_{*} \geq 0$ and $u_{*} \neq 0$, by Lemma 1(ii) and (vi), $\phi_{u_{*}}>0$ and $\phi_{u_{*}} \in C^{0}(\bar{\Omega})$. Therefore, by the strong maximum principle for weak solutions, we obtain that $u_{*}>0$ a.e. in $\Omega$.

(2) We show that $u_{*}$ is a solution of (12); that is, we prove $u_{*}$ satisfying (15) for $\varepsilon=1$.

For given $\delta>0$, define $h:[-\delta, \delta] \rightarrow(-\infty,+\infty)$ by $h(t)=$ $J_{\lambda}\left(u_{*}+t u_{*}\right)$; then $h$ attains its minimum at $t=0$ by Lemma 5 . It implies that

$$
\begin{aligned}
h^{\prime}(0)= & a\left\|u_{*}\right\|^{2}+b\left\|u_{*}\right\|^{4}+\eta \int_{\Omega} \phi_{u_{*}} u_{*}^{2} \\
& -\lambda \int_{\Omega} f(x)\left|u_{*}\right|^{1-\beta}=0 .
\end{aligned}
$$

We take $\varphi \in H_{0}^{1}(\Omega) \backslash\{0\}, \rho>0$ and define $\Psi=\left(u_{*}+\rho \varphi\right)^{+}$. Let

$$
\begin{aligned}
& \Omega_{1}=\left\{x \in \Omega: u_{*}(x)+\rho \varphi(x)>0\right\}, \\
& \Omega_{2}=\left\{x \in \Omega: u_{*}(x)+\rho \varphi(x) \leq 0\right\} .
\end{aligned}
$$

Then $\left.\Psi\right|_{\Omega_{1}}=u_{*}+\rho \varphi,\left.\Psi\right|_{\Omega_{2}}=0$. Inserting $\Psi$ into (26) and using (28), we can obtain that

$$
\begin{aligned}
0 \leq & \int_{\Omega}\left[\left(a+b\left\|u_{*}\right\|^{2}\right) \nabla u_{*} \nabla \Psi+\eta \phi_{u_{*}} u_{*} \Psi\right. \\
& \left.-\lambda f(x) u_{*}^{-\beta} \Psi\right] \\
& =\int_{\Omega_{1}}\left[\left(a+b\left\|u_{*}\right\|^{2}\right) \nabla u_{*} \nabla\left(u_{*}+\rho \varphi\right)\right. \\
& \left.+\eta \phi_{u_{*}} u_{*}\left(u_{*}+\rho \varphi\right)-\lambda f(x) u_{*}^{-\beta}\left(u_{*}+\rho \varphi\right)\right] \\
& =\int_{\Omega \backslash \Omega_{2}}\left[\left(a+b\left\|u_{*}\right\|^{2}\right) \nabla u_{*} \nabla\left(u_{*}+\rho \varphi\right)\right. \\
& \left.+\eta \phi_{u_{*}} u_{*}\left(u_{*}+\rho \varphi\right)-\lambda f(x) u_{*}^{-\beta}\left(u_{*}+\rho \varphi\right)\right] \\
& =\rho \int_{\Omega}\left[\left(a+b\left\|u_{*}\right\|^{2}\right) \nabla u_{*} \nabla \varphi+\eta \phi_{u_{*}} u_{*} \varphi\right. \\
& \left.-\lambda f(x) u_{*}^{-\beta} \varphi\right]-\rho \int_{\Omega_{2}}\left[\left(a+b\left\|u_{*}\right\|^{2}\right) \nabla u_{*} \nabla \varphi\right. \\
& \left.+\eta \phi_{u_{*}} u_{*} \varphi\right] . \\
& \left.+\eta \phi_{u_{*}} u_{*} \varphi-\lambda f(x) u_{*}^{-\beta} \varphi\right] \\
& \leq \rho \int_{\Omega}\left[\left(a+b\left\|u_{*}\right\|^{2}\right) \nabla u_{*} \nabla \varphi+\eta \phi_{u_{*}} u_{*} \varphi\right. \\
& \left.-\lambda f(x) u_{*}^{-\beta} \varphi\right]-\rho \int_{\Omega_{2}}\left[\left(a+b\left\|u_{*}\right\|^{2}\right) \nabla u_{*} \nabla \varphi\right.
\end{aligned}
$$

Since $u_{*}>0$ and the measure of the domain $\Omega_{2}=\{x \in \Omega$ : $\left.u_{*}(x)+\rho \varphi(x) \leq 0\right\}$ tends to zero as $\rho \rightarrow 0$, it follows that

$$
\int_{\Omega_{2}}\left[\left(a+b\left\|u_{*}\right\|^{2}\right) \nabla u_{*} \nabla \varphi+\eta \phi_{u_{*}} u_{*} \varphi\right] \longrightarrow 0
$$

Then dividing by $\rho>0$ and letting $\rho \rightarrow 0$ in (30), we see that

$$
\begin{aligned}
& \int_{\Omega}\left[\left(a+b\left\|u_{*}\right\|^{2}\right) \nabla u_{*} \nabla \varphi+\eta \phi_{u_{*}} u_{*} \varphi-\lambda f(x) u_{*}^{-\beta} \varphi\right] \\
& \quad \geq 0, \quad \varphi \in H_{0}^{1}(\Omega) .
\end{aligned}
$$

This inequality also holds for $-\varphi$, so we get

$$
\begin{aligned}
& \int_{\Omega}\left[\left(a+b\left\|u_{*}\right\|^{2}\right) \nabla u_{*} \nabla \varphi+\eta \phi_{u_{*}} u_{*} \varphi-\lambda f(x) u_{*}^{-\beta} \varphi\right] \\
& =0, \quad \varphi \in H_{0}^{1}(\Omega) .
\end{aligned}
$$


Then $u_{*} \in H_{0}^{1}(\Omega)$ is a solution of (12) for $\varepsilon=1, \lambda>0$, and $\eta \geq 0$.

(3) We show that $u_{*}$ is the unique solution of (12) for $\varepsilon=$ $1, \lambda>0$, and $\eta \geq 0$.

Assume that $v_{*} \in H_{0}^{1}(\Omega)$ is also a solution of (12) for $\varepsilon=$ $1, \lambda>0$, and $\eta \geq 0$. It follows from (15) that

$$
\begin{aligned}
& \left(a+b \int_{\Omega}\left|\nabla u_{*}\right|^{2}\right) \int_{\Omega} \nabla u_{*} \cdot \nabla\left(u_{*}-v_{*}\right) \\
& +\eta \int_{\Omega} \phi_{u_{*}} u_{*}\left(u_{*}-v_{*}\right) \\
& -\lambda \int_{\Omega} f(x) u_{*}^{-\beta}\left(u_{*}-v_{*}\right)=0 . \\
& \left(a+b \int_{\Omega}\left|\nabla v_{*}\right|^{2}\right) \int_{\Omega} \nabla v_{*} \cdot \nabla\left(u_{*}-v_{*}\right) \\
& +\eta \int_{\Omega} \phi_{v_{*}} v_{*}\left(u_{*}-v_{*}\right) \\
& -\lambda \int_{\Omega} f(x) v_{*}^{-\beta}\left(u_{*}-v_{*}\right)=0
\end{aligned}
$$

Subtracting (34) and (35), we obtain that

$$
\begin{aligned}
& a\left\|u_{*}-v_{*}\right\|^{2} \\
& \quad+b\left[\left\|u_{*}\right\|^{4}-\left(\left\|u_{*}\right\|^{2}+\left\|v_{*}\right\|^{2}\right) \int_{\Omega} \nabla u_{*} \nabla v_{*}+\left\|v_{*}\right\|^{4}\right] \\
& \quad+\eta \int_{\Omega}\left(\phi_{u_{*}} u_{*}-\phi_{v_{*}} v_{*}\right)\left(u_{*}-v_{*}\right) \\
& =\lambda \int_{\Omega} f(x)\left(u_{*}^{-\beta}-v_{*}^{-\beta}\right)\left(u_{*}-v_{*}\right) .
\end{aligned}
$$

Since $\beta \in(0,1), u_{*}, v_{*}>0$ in $\Omega$, the following inequality holds:

$$
\lambda \int_{\Omega} f(x)\left(u_{*}^{-\beta}-v_{*}^{-\beta}\right)\left(u_{*}-v_{*}\right) \leq 0
$$

Consequently, it follows from Lemma 1(vii) and (36) that

$$
\left\|u_{*}-v_{*}\right\| \leq 0
$$

which implies that

$$
\left\|u_{*}-v_{*}\right\|=0
$$

that is $\left(u_{*}, \phi_{u_{*}}\right) \in H_{0}^{1}(\Omega) \times H_{0}^{1}(\Omega)$ is the unique solution of system (1).

\section{Proof of Theorem 3}

3.1. The Case of $0 \leq \eta \leq \eta_{0}$. In this part, let $0 \leq \eta \leq \eta_{0}$, where $\eta_{0}=b^{1 / 2} S^{3 / 2}|\Omega|^{-1 / 2}$. From Lemma 1(iv) and (9), we have

$$
\begin{aligned}
J_{\lambda}(u)= & \frac{a}{2}\|u\|^{2}+\frac{b}{4}\|u\|^{4}-\frac{\eta}{4} \int_{\Omega} \phi_{u} u^{2} \\
& -\frac{\lambda}{1-\beta} \int_{\Omega} f(x)|u|^{1-\beta} \\
\geq & \frac{a}{2}\|u\|^{2}+\frac{b}{4}\|u\|^{4}-\frac{\eta^{2}}{4} S^{-3}|\Omega|\|u\|^{4} \\
& -\frac{\lambda}{1-\beta}|f|_{\infty} S^{-(1-\beta) / 2}|\Omega|^{(5+\beta) / 6}\|u\|^{1-\beta} \\
\geq & \frac{a}{2}\|u\|^{2} \\
& -\frac{\lambda}{1-\beta}|f|_{\infty} S^{-(1-\beta) / 2}|\Omega|^{(5+\beta) / 6}\|u\|^{1-\beta},
\end{aligned}
$$

$\forall u \in H_{0}^{1}(\Omega)$

Thus, $J_{\lambda}(u)$ is coercive and bounded from below on $H_{0}^{1}(\Omega)$ for all $0 \leq \eta \leq \eta_{0}$ and $\lambda>0$. So

$$
\alpha_{\lambda}=\inf _{u \in H_{0}^{1}(\Omega)} J_{\lambda}(u)
$$

is well defined. Obviously, $\alpha_{\lambda}<0$. Similar to the proof of Lemma 5 , we easily obtain the following proposition.

Proposition 6. For all $a>0, b \geq 0, \varepsilon=-1$, and $\beta \in(0,1)$, the functional $J_{\lambda}$ attains the global minimizer in $H_{0}^{1}(\Omega)$; that is, there exists $u^{*} \in H_{0}^{1}(\Omega)$ such that

$$
J_{\lambda}\left(u^{*}\right)=\alpha_{\lambda}=\inf _{u \in H_{0}^{1}(\Omega)} J_{\lambda}(u)<0 .
$$

Proof of Theorem 3(i). The proof is similar to parts (1) and (2) of the proof in Theorem 2, so we omit it.

3.2. The Case of $\eta>\eta_{0}$. In this part, let $\eta>\eta_{0} . J_{\lambda}(u)$ is not necessarily coercive and bounded from below on $H_{0}^{1}(\Omega)$ for $\eta>\eta_{0}$. We define a Nehari manifold $N_{\lambda}$ by

$$
\begin{aligned}
N_{\lambda} & =\left\{u \in H_{0}^{1}(\Omega): a\|u\|^{2}+b\|u\|^{4}-\eta \int_{\Omega} \phi_{u} u^{2}\right. \\
& \left.-\lambda \int_{\Omega} f(x)|u|^{1-\beta} d x=0\right\} .
\end{aligned}
$$


Then the solutions of (12) must lie in $N_{\lambda}$. Obviously, $N_{\lambda}$ is a closed set in $H_{0}^{1}(\Omega)$. In order to obtain the multiplicity, we split $N_{\lambda}$ into the following three parts:

$$
\begin{aligned}
N_{\lambda}^{+} & =\left\{u \in N_{\lambda}: 2 a\|u\|^{2}+4 b\|u\|^{4}-4 \eta \int_{\Omega} \phi_{u} u^{2}\right. \\
& \left.-\lambda(1-\beta) \int_{\Omega} f(x)|u|^{1-\beta} d x>0\right\}, \\
N_{\lambda}^{0} & =\left\{u \in N_{\lambda}: 2 a\|u\|^{2}+4 b\|u\|^{4}-4 \eta \int_{\Omega} \phi_{u} u^{2}\right. \\
& \left.-\lambda(1-\beta) \int_{\Omega} f(x)|u|^{1-\beta} d x=0\right\}, \\
N_{\lambda}^{-} & =\left\{u \in N_{\lambda}: 2 a\|u\|^{2}+4 b\|u\|^{4}-4 \eta \int_{\Omega} \phi_{u} u^{2}\right. \\
& \left.-\lambda(1-\beta) \int_{\Omega} f(x)|u|^{1-\beta} d x<0\right\} .
\end{aligned}
$$

When $u \in N_{\lambda}$, we have

$$
\begin{aligned}
& 2 a\|u\|^{2}+4 b\|u\|^{4}-4 \eta \int_{\Omega} \phi_{u} u^{2} \\
& \quad-\lambda(1-\beta) \int_{\Omega} f(x)|u|^{1-\beta}=-2 a\|u\|^{2} \\
& \quad+(3+\beta) \lambda \int_{\Omega} f(x)|u|^{1-\beta}=2 b\|u\|^{4}-2 \eta \int_{\Omega} \phi_{u} u^{2} \\
& \quad+(1+\beta) \lambda \int_{\Omega} f(x)|u|^{1-\beta}=(1+\beta) a\|u\|^{2} \\
& \quad+(3+\beta) b\|u\|^{4}-(3+\beta) \eta \int_{\Omega} \phi_{u} u^{2} .
\end{aligned}
$$

Lemma 7. For any $\eta>\eta_{0}$ there exists $\lambda_{*}>0$ such that $N_{\lambda}^{ \pm} \neq \emptyset$ and $N_{\lambda}^{0}=\{0\}$ for $\lambda \in\left(0, \lambda_{*}\right)$.

Proof. For any given $u \in H_{0}^{1}(\Omega) \backslash\{0\}, t \geq 0$, by calculating, we can get that

$$
\begin{aligned}
& t \frac{d}{d t}\left[J_{\lambda}(t u)\right]=a t^{2}\|u\|^{2}+b t^{4}\|u\|^{4}-\eta t^{4} \int_{\Omega} \phi_{u} u^{2} \\
& -\lambda t^{1-\beta} \int_{\Omega} f(x)|u|^{1-\beta}=t^{1-\beta}\left(a t^{1+\beta}\|u\|^{2}\right. \\
& \left.+b t^{3+\beta}\|u\|^{4}-\eta t^{3+\beta} \int_{\Omega} \phi_{u} u^{2}-\lambda \int_{\Omega} f(x)|u|^{1-\beta}\right) .
\end{aligned}
$$

Let

$$
\Phi_{u}(t)=a t^{1+\beta}\|u\|^{2}+b t^{3+\beta}\|u\|^{4}-\eta t^{3+\beta} \int_{\Omega} \phi_{u} u^{2},
$$

$$
t \geq 0 \text {. }
$$

Then,

$$
\begin{aligned}
\Phi_{u}^{\prime}(t)= & (1+\beta) a t^{\beta}\|u\|^{2}+(3+\beta) b t^{2+\beta}\|u\|^{4} \\
& -(3+\beta) \eta t^{2+\beta} \int_{\Omega} \phi_{u} u^{2} .
\end{aligned}
$$

Since $\eta>\eta_{0}$, it is easy to obtain that

$$
G=\left\{u \in H_{0}^{1}(\Omega) \mid b\|u\|^{4}-\eta \int_{\Omega} \phi_{u} u^{2}<0\right\} \neq \emptyset .
$$

Therefore, for any $u \in G$, let $\Phi_{u}^{\prime}(t)=0$. Then

$$
t_{u}=\left[\frac{a(1+\beta)\|u\|^{2}}{(3+\beta)\left(\eta \int_{\Omega} \phi_{u} u^{2}-b\|u\|^{4}\right)}\right]^{1 / 2} .
$$

Moreover, $\Phi_{u}^{\prime}(t)>0$ for all $0<t<t_{u}$ and $\Phi_{u}^{\prime}(t)<0$ for all $t>t_{u}$. Thus $\Phi_{u}$ is increasing for all $0<t<t_{u}$ and decreasing for all $t>t_{u}$. So

$$
\begin{aligned}
& \max _{t \in[0,+\infty)} \Phi_{u}(t)=\Phi_{u}\left(t_{u}\right)=\frac{2 a}{3+\beta}\left[\frac{a(1+\beta)}{3+\beta}\right]^{(1+\beta) / 2} \\
& \cdot \frac{\|u\|^{3+\beta}}{\left(\eta \int_{\Omega} \phi_{u} u^{2}-b\|u\|^{4}\right)^{(1+\beta) / 2}}>0 \\
& \Phi_{u}(0)=0 \\
& \lim _{t \rightarrow \infty} \Phi_{u}(t)=-\infty
\end{aligned}
$$

Thus from Lemma 1(iv), (9), and (53), it follows that

$$
\begin{gathered}
\Phi_{u}\left(t_{u}\right)-\lambda \int_{\Omega} f(x)|u|^{1-\beta} d x \\
\geq \frac{2 a}{3+\beta}\left[\frac{a(1+\beta)}{3+\beta}\right]^{(1+\beta) / 2} \\
\cdot \frac{\|u\|^{3+\beta}}{\left(\eta \int_{\Omega} \phi_{u} u^{2}-b\|u\|^{4}\right)^{(1+\beta) / 2}}-\lambda|f|_{\infty} \\
\cdot S^{-(1-\beta) / 2}|\Omega|^{(5+\beta) / 6}\|u\|^{1-\beta} \\
\geq\left\{\frac{2 a}{3+\beta}\left[\frac{a(1+\beta)}{3+\beta}\right]^{(1+\beta) / 2}\right. \\
\cdot \frac{1}{\left(\eta^{2} S^{-3}|\Omega|-b\right)^{(1+\beta) / 2}-\lambda|f|_{\infty}} \\
\left.\cdot S^{-(1-\beta) / 2}|\Omega|^{(5+\beta) / 6}\right\}\|u\|^{1-\beta}>0
\end{gathered}
$$

for all $0<\lambda<\lambda_{*}$.

From (54) and (55), we obtain that there exist unique positive numbers $t_{u}^{+}<t_{u}<t_{u}^{-}$such that

$$
\begin{aligned}
& \Phi_{u}\left(t_{u}^{+}\right)=\lambda \int_{\Omega} f(x)|u|^{1-\beta} d x=\Phi_{u}\left(t_{u}^{-}\right), \\
& \Phi_{u}^{\prime}\left(t_{u}^{+}\right)>0 \\
& \Phi_{u}^{\prime}\left(t_{u}^{-}\right)<0 .
\end{aligned}
$$

From (48)-(50), it follows that $t_{u}^{+} u \in N_{\lambda}^{+}$and $t_{u}^{-} u \in N_{\lambda}^{-}$for all $0<\lambda<\lambda_{*}$. 
Next, we prove that $N_{\lambda}^{0}=\{0\}$ for all $0<\lambda<\lambda_{*}$. Assume that there exists $u_{0} \in N_{\lambda}^{0}$ and $u_{0} \neq 0$. Then it follows that

$$
\begin{aligned}
a & (1+\beta)\left\|u_{0}\right\|^{2}+b(3+\beta)\left\|u_{0}\right\|^{4} \\
& -(3+\beta) \eta \int_{\Omega} \phi_{u_{0}}\left(u_{0}\right)^{2}=0, \\
0 & =a\left\|u_{0}\right\|^{2}+b\left\|u_{0}\right\|^{4}-\eta \int_{\Omega} \phi_{u_{0}} u_{0}^{2} \\
& -\lambda \int_{\Omega} f(x)\left|u_{0}\right|^{1-\beta} d x=\frac{2 a}{3+\beta}\left\|u_{0}\right\|^{2} \\
& -\lambda \int_{\Omega} f(x)\left|u_{0}\right|^{1-\beta} d x .
\end{aligned}
$$

From (57) and (55), one has $u_{0} \in G$ and

$$
\begin{aligned}
0 & <\frac{2 a}{3+\beta}\left[\frac{a(1+\beta)}{3+\beta}\right]^{(1+\beta) / 2} \\
& \cdot \frac{\left\|u_{0}\right\|^{3+\beta}}{\left(\eta \int_{\Omega} \phi_{u_{0}} u_{0}^{2}-b\left\|u_{0}\right\|^{4}\right)^{(1+\beta) / 2}}-\lambda|f|_{\infty} \\
& \cdot S^{-(1-\beta) / 2}|\Omega|^{(5+\beta) / 6}\left\|u_{0}\right\|^{1-\beta} \\
& \leq \frac{2 a}{3+\beta}\left[\frac{a(1+\beta)}{3+\beta}\right]^{(1+\beta) / 2} \\
& \cdot \frac{\left\|u_{0}\right\|^{3+\beta}}{\left(\eta \int_{\Omega} \phi_{u_{0}} u_{0}^{2}-b\left\|u_{0}\right\|^{4}\right)^{(1+\beta) / 2}} \\
& -\lambda \int_{\Omega} f(x)\left|u_{0}\right|^{1-\beta} d x \\
& =\frac{2 a}{3+\beta}\left[\frac{a(1+\beta)}{3+\beta}\right]^{(1+\beta) / 2} \\
& \cdot \frac{\left\|u_{0}\right\|^{3+\beta}}{\left((a(1+\beta) /(3+\beta))\left\|u_{0}\right\|^{2}\right)^{(1+\beta) / 2}} \\
& -\frac{2 a}{3+\beta}\left\|u_{0}\right\|^{2}=0
\end{aligned}
$$

for all $0<\lambda<\lambda_{*}$, which implies a contradiction. Thus $N_{\lambda}^{0}=$ $\{0\}$ for all $0<\lambda<\lambda_{*}$.

Lemma 8. $N_{\lambda}^{-}$is a closed set in $H_{0}^{1}(\Omega)$ for $\lambda \in\left(0, \lambda_{*}\right)$.
Proof. Suppose $u_{n} \subset N_{\lambda}^{-}$such that $u_{n} \rightarrow u$ in $H_{0}^{1}(\Omega)$ as $n \rightarrow$ $\infty$. From the definition of $N_{\lambda}^{-}$, one has

$$
\begin{gathered}
a\left\|u_{n}\right\|^{2}+b\left\|u_{n}\right\|^{4}-\eta \int_{\Omega} \phi_{u_{n}} u_{n}^{2} \\
-\lambda \int_{\Omega} f(x)\left|u_{n}\right|^{1-\beta} d x=0, \\
a(1+\beta)\left\|u_{n}\right\|^{2}+b(3+\beta)\left\|u_{n}\right\|^{4} \\
-\eta(3+\beta) \int_{\Omega} \phi_{u_{n}} u_{n}^{2}<0 .
\end{gathered}
$$

Thus it follows from $u_{n} \rightarrow u$ in $H_{0}^{1}(\Omega)$ as $n \rightarrow \infty$ that

$$
\begin{aligned}
& a\|u\|^{2}+b\|u\|^{4}-\eta \int_{\Omega} \phi_{u} u^{2}-\lambda \int_{\Omega} f(x)|u|^{1-\beta} d x \\
& \quad=0, \\
& a(1+\beta)\|u\|^{2}+b(3+\beta)\|u\|^{4}-\eta(3+\beta) \int_{\Omega} \phi_{u} u^{2}
\end{aligned}
$$$$
\leq 0,
$$

so $u \in N_{\lambda}^{0} \cup N_{\lambda}^{-}$. If $u \in N_{\lambda}^{0}$, then $u=0$. However, from (60), we obtain

$$
\|u\|^{2} \geq \frac{a(1+\beta)}{(3+\beta)\left(\eta^{2} S^{-3}|\Omega|-b\right)}, \quad \forall u_{n} \in N_{\lambda}^{-},
$$

which contracts $u=0$. Thus $u \in N_{\lambda}^{-}$for all $0<\lambda<\lambda_{*}$. Therefore, $N_{\lambda}^{-}$is closed for all $0<\lambda<\lambda_{*}$.

Lemma 9. Assume $\eta>\eta_{0}$ and $\lambda \in\left(0, \lambda_{*}\right), J_{\lambda}(u)$ is coercive and bounded from below on $N_{\lambda}$ and $\inf _{u \in N_{\lambda}^{+} \cup N_{\lambda}^{0}} J_{\lambda}(u)<0$.

Proof. For all $u \in N_{\lambda}$, from (9) we have

$$
\begin{aligned}
J_{\lambda}(u) & \\
= & \frac{a}{2}\|u\|^{2}+\frac{b}{4}\|u\|^{4}-\frac{\eta}{4} \int_{\Omega} \phi_{u} u^{2} \\
& -\frac{\lambda}{1-\beta} \int_{\Omega} f(x)|u|^{1-\beta} \\
= & \frac{a}{2}\|u\|^{2}+\frac{1}{4}\left(-a\|u\|^{2}+\lambda \int_{\Omega} f(x)|u|^{1-\beta} d x\right) \\
& -\frac{\lambda}{1-\beta} \int_{\Omega} f(x)|u|^{1-\beta} \\
= & \frac{a}{4}\|u\|^{2}-\lambda\left(\frac{1}{1-\beta}-\frac{1}{4}\right) \int_{\Omega} f(x)|u|^{1-\beta} \\
\geq & \frac{a}{4}\|u\|^{2} \\
& -\lambda\left(\frac{1}{1-\beta}-\frac{1}{4}\right)|f|_{\infty} S^{-(1-\beta) / 2}|\Omega|^{(5+\beta) / 6}\|u\|^{1-\beta} .
\end{aligned}
$$

It follows from $0<\beta<1$ that $J_{\lambda}(u)$ is coercive and bounded from below on $N_{\lambda}$ for any $\lambda \in\left(0, \lambda_{*}\right)$. 
Since $N_{\lambda}^{+} \cup N_{\lambda}^{0}$ and $N_{\lambda}^{-}$are two nonempty closed subsets of $H_{0}^{1}(\Omega)$ for all $\eta>\eta_{0}$ and $\lambda \in\left(0, \lambda_{*}\right)$, it follows that $\alpha_{\lambda}^{+}=$ $\inf _{u \in N_{\lambda}^{+} \cup N_{\lambda}^{0}} J_{\lambda}(u)$ and $\alpha_{\lambda}^{-}=\inf _{u \in N_{\lambda}^{-}} J_{\lambda}(u)$ are well defined. For any given $u \in N_{\lambda}^{+}$, by (47), we have

$$
a(1+\beta)\|u\|^{2}+b(3+\beta)\|u\|^{4}-\eta(3+\beta) \int_{\Omega} \phi_{u} u^{2}
$$

$>0$.

Consequently,

$J_{\lambda}(u)$

$$
\begin{aligned}
= & \frac{a}{2}\|u\|^{2}+\frac{b}{4}\|u\|^{4}-\frac{\eta}{4} \int_{\Omega} \phi_{u} u^{2} \\
& -\frac{\lambda}{1-\beta} \int_{\Omega} f(x)|u|^{1-\beta} \\
= & -\frac{a(1+\beta)}{2(1-\beta)}\|u\|^{2}-\frac{b(3+\beta)}{4(1-\beta)}\|u\|^{4} \\
& +\frac{\eta(3+\beta)}{4(1-\beta)} \int_{\Omega} \phi_{u} u^{2} \\
< & \frac{-a(1+\beta)\|u\|^{2}-b(3+\beta)\|u\|^{4}+\eta(3+\beta) \int_{\Omega} \phi_{u} u^{2}}{4(1-\beta)}
\end{aligned}
$$

$<0$.

Thus, by Lemma 7, $\alpha_{\lambda}^{+}=\inf _{u \in N_{\lambda}^{+} \cup N_{\lambda}^{0}} J_{\lambda}(u)<0$ for all $\eta>\eta_{0}$ and $0<\lambda<\lambda_{*}$.

Lemma 10. Assume that $\eta>\eta_{0}$ and $\lambda \in\left(0, \lambda_{*}\right)$, given $u \in$ $N_{\lambda}^{+}$(resp., $N_{\lambda}^{-}$), there exists $\varepsilon>0$ and a continuous function $f=f(w)>0, w \in H_{0}^{1}(\Omega),\|w\|<\varepsilon$, satisfying that

$$
\begin{aligned}
f(0) & =1, \\
f(w)(u+w) & \in N_{\lambda}^{+}\left(\text {resp. } N_{\lambda}^{-}\right),
\end{aligned}
$$

$$
\forall w \in H_{0}^{1}(\Omega),\|w\|<\varepsilon .
$$

Proof. Please see Lemma 3.5 of [1] for the similar proof.

Proof of Theorem 3(ii). By Ekeland's variational principle, there exists a minimizing sequence $\left\{u_{n}\right\} \subset N_{\lambda}^{+} \cup N_{\lambda}^{0}$ satisfying

(i) $J_{\lambda}\left(u_{n}\right) \leq \inf _{u \in N_{\lambda}^{+} \cup N_{\lambda}^{0}} J_{\lambda}(u)+1 / n^{2}$,

(ii) $J_{\lambda}(u) \geq J_{\lambda}\left(u_{n}\right)-(1 / n)\left\|u-u_{n}\right\|, u \in N_{\lambda}^{+} \cup N_{\lambda}^{0}$.

From $J_{\lambda}(|u|)=J_{\lambda}(u)$ and Lemma 9 , we may assume that $u_{n} \in N_{\lambda}^{+}$and $u_{n} \geq 0$. It is easy to obtain that $\left\{u_{n}\right\}$ is bounded in $H_{0}^{1}(\Omega)$; we assume that $\left\|u_{n}\right\| \leq C_{0}$. Going if necessary to a subsequence, we can assume that

$$
\begin{aligned}
u_{n} & \rightarrow u_{0}, \quad \text { in } H_{0}^{1}(\Omega) \\
u_{n} & \longrightarrow u_{0}, \quad \text { in } L^{p}(\Omega), p \in[1,6) \\
u_{n}(x) & \longrightarrow u_{0}(x), \quad \text { a.e. in } \Omega .
\end{aligned}
$$

Since $\left\{u_{n}\right\} \subset N_{\lambda}^{+} \subset N_{\lambda}$, we get

$$
\begin{aligned}
a\left\|u_{n}\right\|^{2} & +b\left\|u_{n}\right\|^{4}-\eta \int_{\Omega} \phi_{u_{n}} u_{n}^{2} \\
& -\lambda \int_{\Omega} f(x)\left|u_{n}\right|^{1-\beta} d x=0 .
\end{aligned}
$$

By the weakly lower semicontinuity of the norm, Lemma 1(v), (14), and (47), we have

$$
\begin{aligned}
& a\left\|u_{0}\right\|^{2}+b\left\|u_{0}\right\|^{4}-\eta \int_{\Omega} \phi_{u_{0}} u_{0}^{2}-\lambda \int_{\Omega} f(x)\left|u_{0}\right|^{1-\beta} d x \\
& \quad \leq 0, \\
& -2 a\left\|u_{0}\right\|^{2}+(3+\beta) \lambda \int_{\Omega} f(x)\left|u_{0}\right|^{1-\beta} d x \geq 0, \\
& J_{\lambda}\left(u_{0}\right) \leq \lim _{n \rightarrow \infty} J_{\lambda}\left(u_{n}\right)=\inf _{u \in N_{\lambda}^{+} \cup N_{\lambda}^{0}} J_{\lambda}(u)<0 .
\end{aligned}
$$

Therefore, $u_{0}(x) \geq 0$ and $u_{0}(x) \not \equiv 0$.

First, we prove that $u_{0}(x)>0$ in $\Omega$. Since $\left\{u_{n}\right\} \subset N_{\lambda}^{+}$, we can claim that there exists a constant $C_{1}>0$ such that up to a subsequence we have

$$
\begin{aligned}
& a(1+\beta)\left\|u_{n}\right\|^{2}+b(3+\beta)\left\|u_{n}\right\|^{4}-\eta(3+\beta) \int_{\Omega} \phi_{u_{n}} u_{n}^{2} \\
& \quad \geq C_{1} .
\end{aligned}
$$

In order to prove (70), it suffices to verify

$$
\begin{aligned}
& a(1+\beta) \varlimsup_{n \rightarrow \infty}\left\|u_{n}\right\|^{2}+b(3+\beta) \varlimsup_{n \rightarrow \infty}\left\|u_{n}\right\|^{4} \\
& >\eta(3+\beta) \int_{\Omega} \phi_{u_{0}} u_{0}^{2} .
\end{aligned}
$$

Since $u_{n} \in N_{\lambda}^{+}$,

$$
a(1+\beta)\left\|u_{n}\right\|^{2}+b(3+\beta)\left\|u_{n}\right\|^{4}-\eta(3+\beta) \int_{\Omega} \phi_{u_{n}} u_{n}^{2}
$$

$>0$.

It follows that

$$
\begin{aligned}
& a(1+\beta) \varlimsup_{n \rightarrow \infty}\left\|u_{n}\right\|^{2}+b(3+\beta) \varlimsup_{n \rightarrow \infty}\left\|u_{n}\right\|^{4} \\
& \geq \eta(3+\beta) \int_{\Omega} \phi_{u_{0}} u_{0}^{2} .
\end{aligned}
$$

By contradiction, we assume that

$$
\begin{aligned}
& a(1+\beta) \varlimsup_{n \rightarrow \infty}\left\|u_{n}\right\|^{2}+b(3+\beta) \varlimsup_{n \rightarrow \infty}\left\|u_{n}\right\|^{4} \\
& \quad=\eta(3+\beta) \int_{\Omega} \phi_{u_{0}} u_{0}^{2} .
\end{aligned}
$$

From the boundedness of $\left\{u_{n}\right\}$ and (72), one has

$$
\begin{aligned}
& a(1+\beta) \underset{n \rightarrow \infty}{\varliminf_{n \rightarrow \infty}}\left\|u_{n}\right\|^{2}+b(3+\beta) \varliminf_{n \rightarrow \infty}\left\|u_{n}\right\|^{4} \\
& \geq \eta(3+\beta) \int_{\Omega} \phi_{u_{0}} u_{0}^{2},
\end{aligned}
$$


which combines with (74); it follows that

$$
\begin{aligned}
& a(1+\beta) \lim _{n \rightarrow \infty}\left\|u_{n}\right\|^{2}+b(3+\beta) \lim _{n \rightarrow \infty}\left\|u_{n}\right\|^{4} \\
& =\eta(3+\beta) \int_{\Omega} \phi_{u_{0}} u_{0}^{2} .
\end{aligned}
$$

Let $\lim _{n \rightarrow \infty}\left\|u_{n}\right\|=\ell$; then $\ell>0$ and

$$
a(1+\beta) \ell+b(3+\beta) \ell^{2}=\eta(3+\beta) \int_{\Omega} \phi_{u_{0}} u_{0}^{2} .
$$

Since $u_{n} \in N_{\lambda}$, one has

$$
a \ell+b \ell^{2}-\eta \int_{\Omega} \phi_{u_{0}} u_{0}^{2}-\lambda \int_{\Omega} f(x)\left|u_{0}\right|^{1-\beta} d x=0
$$

Consequently, we have

$$
\frac{2 a \ell}{3+\beta}=\lambda \int_{\Omega} f(x)\left|u_{0}\right|^{1-\beta} d x .
$$

For all $0<\lambda<\lambda_{*}$, from (55), (77), and (79),

$$
\begin{aligned}
0< & \frac{2 a}{3+\beta}\left[\frac{a(1+\beta)}{3+\beta}\right]^{(1+\beta) / 2} \\
& \cdot \frac{\left\|u_{0}\right\|^{3+\beta}}{\left(\eta \int_{\Omega} \phi_{u_{0}} u_{0}^{2}-b\left\|u_{0}\right\|^{4}\right)^{(1+\beta) / 2}}-\lambda|f|_{\infty} \\
& \cdot S^{-(1-\beta) / 2}|\Omega|^{(5+\beta) / 6}\left\|u_{0}\right\|^{1-\beta} \\
& <\frac{2 a}{3+\beta}\left[\frac{a(1+\beta)}{3+\beta}\right]^{(1+\beta) / 2} \\
& \cdot \frac{\left\|u_{0}\right\|^{3+\beta}}{\left(\eta \int_{\Omega} \phi_{u_{0}} u_{0}^{2}-b\left\|u_{0}\right\|^{4}\right)^{(1+\beta) / 2}} \\
& -\lambda \int_{\Omega} f(x)\left|u_{0}\right|^{1-\beta} d x \\
& =\frac{2 a}{3+\beta}\left[\frac{a(1+\beta)}{3+\beta}\right]^{(1+\beta) / 2} \\
& \cdot \frac{\ell^{(3+\beta) / 2}}{((a(1+\beta) /(3+\beta)) \ell)^{(1+\beta) / 2}}-\frac{2 a \ell}{3+\beta}=0
\end{aligned}
$$

which is a contradiction. Thus our claim is true; that is, (70) and (71) must hold.

Taking $N=2(1-\beta) C_{0} C_{1} /(1+\beta)$, for fixed $\varphi \in H_{0}^{1}(\Omega)$ and $\varphi>0$ in $\Omega$, we apply Lemma 10 with $u=u_{n}$, and $w=$ $s \varphi, s>0$, small enough; it is easy to see that $g_{n}(s)=f_{n}(s \varphi)$ such that $g_{n}(0)=1$ and $g_{n}\left(u_{n}+s \varphi\right) \in N_{\lambda}^{+}$. It follows from $u_{n}, g_{n}(s)\left(u_{n}+s \varphi\right) \in N_{\lambda}^{+} \subset N_{\lambda}$ that

$$
\begin{aligned}
& a\left\|u_{n}\right\|^{2}+b\left\|u_{n}\right\|^{4}-\eta \int_{\Omega} \phi_{u_{n}} u_{n}^{2} \\
& -\lambda \int_{\Omega} f(x)\left|u_{n}\right|^{1-\beta} d x=0, \\
& a g_{n}^{2}(s)\left\|u_{n}+s \varphi\right\|^{2}+b g_{n}^{4}(s)\left\|u_{n}+s \varphi\right\|^{4} \\
& \quad-\eta g_{n}^{4}(s) \int_{\Omega} \phi_{u_{n}+s \varphi}\left(u_{n}+s \varphi\right)^{2} \\
& -\lambda g_{n}^{1-\beta}(s) \int_{\Omega} f(x)\left|u_{n}+s \varphi\right|^{1-\beta} d x=0 .
\end{aligned}
$$

By the above equalities, we have

$$
\begin{aligned}
0= & \left(g_{n}^{2}(s)-1\right) a\left\|u_{n}+s \varphi\right\|^{2} \\
& +a\left(\left\|u_{n}+s \varphi\right\|^{2}-\left\|u_{n}\right\|^{2}\right) \\
& +\left(g_{n}^{4}(s)-1\right) b\left\|u_{n}+s \varphi\right\|^{4} \\
& +b\left(\left\|u_{n}+s \varphi\right\|^{4}-\left\|u_{n}\right\|^{4}\right) \\
& -\left(g_{n}^{4}(s)-1\right) \eta \int_{\Omega} \phi_{u_{n}+s \varphi}\left(u_{n}+s \varphi\right)^{2} \\
& -\eta \int_{\Omega} \phi_{u_{n}+s \varphi}^{2}\left(u_{n}+s \varphi\right)^{2}-\phi_{u_{n}} u_{n}^{2} d x \\
& -\left(g_{n}^{1-\beta}(s)-1\right) \lambda \int_{\Omega} f(x)\left|u_{n}+s \varphi\right|^{1-\beta} d x \\
& -\lambda \int_{\Omega} f(x)\left(\left|u_{n}+s \varphi\right|^{1-\beta}-\left|u_{n}\right|^{1-\beta}\right) d x \\
\leq & \left(g_{n}^{2}(s)-1\right) a\left\|u_{n}+s \varphi\right\|^{2} \\
& +a\left(\left\|u_{n}+s \varphi\right\|^{2}-\left\|u_{n}\right\|^{2}\right) \\
& +\left(g_{n}^{4}(s)-1\right) b\left\|u_{n}+s \varphi\right\|^{4} \\
& +b\left(\left\|u_{n}+s \varphi\right\|^{4}-\left\|u_{n}\right\|^{4}\right) \\
& -\left(g_{n}^{4}(s)-1\right) \eta \int_{\Omega} \phi_{u_{n}+s \varphi}\left(u_{n}+s \varphi\right)^{2} \\
& -\eta \int_{\Omega} \phi_{u_{n}+s \varphi}^{2}\left(u_{n}+s \varphi\right)^{2}-\phi_{u_{n}} u_{n}^{2} d x \\
& -\left(g_{n}^{1-\beta}(s)-1\right) \lambda \int_{\Omega} f(x)\left|u_{n}+s \varphi\right|^{1-\beta} d x .
\end{aligned}
$$

Denote $D^{+} g_{n}(0)$ the right upper Dini derivative of $g_{n}$ at zero. Then, dividing by $s>0$ and letting $s \rightarrow 0^{+}$, we deduce that

$$
\begin{aligned}
0 \leq & 2 a D^{+} g_{n}(0)\left\|u_{n}\right\|^{2}+2 a \int_{\Omega} \nabla u_{n} \nabla \phi d x \\
& +4 b D^{+} g_{n}(0)\left\|u_{n}\right\|^{4}+4 b\left\|u_{n}\right\|^{2} \int_{\Omega} \nabla u_{n} \nabla \varphi d x
\end{aligned}
$$




$$
\begin{aligned}
& -4 \eta D^{+} g_{n}(0) \int_{\Omega} \phi_{u_{n}} u_{n}^{2}-4 \eta \int_{\Omega} \phi_{u_{n}} u_{n}^{2}-(1-\beta) \\
& \cdot \lambda D^{+} g_{n}(0) \int_{\Omega} f(x) u_{n}^{1-\beta} d x=D^{+} g_{n}(0)\left[2 a\left\|u_{n}\right\|^{2}\right. \\
& +4 b\left\|u_{n}\right\|^{4}-4 \eta \int_{\Omega} \phi_{u_{n}} u_{n}^{2} \\
& \left.-(1-\beta) \lambda \int_{\Omega} f(x) u_{n}^{1-\beta} d x\right]+\left[2 a \int_{\Omega} \nabla u_{n} \nabla \varphi d x\right. \\
& \left.+4 b\left\|u_{n}\right\|^{2} \int_{\Omega} \nabla u_{n} \nabla \varphi d x-4 \eta \int_{\Omega} \phi_{u_{n}} u_{n}^{2}\right] \\
& =D^{+} g_{n}(0)\left[(1+\beta) a\left\|u_{n}\right\|^{2}+(3+\beta) b\left\|u_{n}\right\|^{4}\right. \\
& \left.-(3+\beta) \eta \int_{\Omega} \phi_{u_{n}} u_{n}^{2}\right]+\left[2 a \int_{\Omega} \nabla u_{n} \nabla \varphi d x\right. \\
& \left.+4 b\left\|u_{n}\right\|^{2} \int_{\Omega} \nabla u_{n} \nabla \varphi d x-4 \eta \int_{\Omega} \phi_{u_{n}} u_{n}^{2}\right]
\end{aligned}
$$

By $\left\|u_{n}\right\| \leq C_{0}$ and Lemma 1(iv), there exist $C_{2}, C_{3}>0$ independent of $n$ such that

$$
\begin{aligned}
& \mid 2 a \int_{\Omega} \nabla u_{n} \nabla \varphi d x+4 b\left\|u_{n}\right\|^{2} \int_{\Omega} \nabla u_{n} \nabla \varphi d x \\
& \quad-4 \eta \int_{\Omega} \phi_{u_{n}} u_{n}^{2} \mid \leq C_{2}, \\
& \quad \mid \frac{1}{1-\beta}\left[(1+\beta) a \int_{\Omega} \nabla u_{n} \nabla \varphi\right. \\
& \quad+(3+\beta) b\left\|u_{n}\right\|^{2} \int_{\Omega} \nabla u_{n} \nabla \varphi \\
& \left.\quad-(3+\beta) \eta \int_{\Omega} \phi_{u_{n}} u_{n} \varphi\right] \mid \leq C_{3} .
\end{aligned}
$$

It follows from (70), (83), and (84) that

$$
D^{+} g_{n}(0) \geq-C_{1}^{-1} C_{2}
$$

In the following part, we prove

$$
D^{+} g_{n}(0) \leq 2\left(\|\varphi\|+C_{3}\right) C_{1}^{-1}, \quad \forall n \geq N
$$

Fixing $n \geq N$, without loss of generality, we may assume $D^{+} g_{n}(0) \geq 0$. Thus, from condition (ii) and (65), we have

$$
\begin{aligned}
& \frac{\left|g_{n}(s)-1\right| \cdot\left\|u_{n}\right\|}{n}+\frac{\left|s g_{n}(s)\right| \cdot\|\varphi\|}{n} \\
& \geq \frac{1}{n}\left\|g_{n}(s)\left(u_{n}+s \varphi\right)-u_{n}\right\| \\
& \geq J_{\lambda}\left(u_{n}\right)-J_{\lambda}\left[g_{n}(s)\left(u_{n}+s \varphi\right)\right] \\
& =\frac{1+\beta}{2(1-\beta)}\left(a\left\|u_{n}+s \varphi\right\|^{2}-a\left\|u_{n}\right\|^{2}\right) \\
& \quad+\frac{1+\beta}{2(1-\beta)} a\left[g_{n}^{2}(s)-1\right]\left\|u_{n}+s \varphi\right\|^{2} \\
& \quad+\frac{3+\beta}{4(1-\beta)}\left(b\left\|u_{n}+s \varphi\right\|^{4}-b\left\|u_{n}\right\|^{4}\right) \\
& \quad+\frac{3+\beta}{4(1-\beta)} b\left[g_{n}^{4}(s)-1\right]\left\|u_{n}+s \varphi\right\|^{4} \\
& \quad-\eta \frac{3+\beta}{4(1-\beta)} g_{n}^{4}(s) \int_{\Omega}\left[\phi_{u_{n}+s \varphi}\left(u_{n}+s \varphi\right)^{2}-\phi_{u_{n}} u_{n}^{2}\right] \\
& \quad-\eta \frac{3+\beta}{4(1-\beta)}\left[g_{n}^{4}(s)-1\right] \int_{\Omega} \phi_{u_{n}} u_{n}^{2} d x .
\end{aligned}
$$

Dividing by $s>0$ and letting $s \rightarrow 0^{+}$, we derive that

$$
\begin{aligned}
D^{+} g_{n}(0) \frac{\left\|u_{n}\right\|}{n}+\frac{\|\varphi\|}{n} \\
\geq \frac{a(1+\beta)}{1-\beta} \int_{\Omega} \nabla u_{n} \nabla \varphi+\frac{a(1+\beta)}{1-\beta} D^{+} g_{n}(0)\left\|u_{n}\right\|^{2} \\
\quad+\frac{b(3+\beta)}{1-\beta}\left\|u_{n}\right\|^{2} \int_{\Omega} \nabla u_{n} \nabla \varphi \\
\quad+\frac{b(3+\beta)}{1-\beta} D^{+} g_{n}(0)\left\|u_{n}\right\|^{4} \\
\quad-\frac{\eta(3+\beta)}{1-\beta} \int_{\Omega} \phi_{u_{n}} u_{n} \phi \\
\quad-\frac{\eta(3+\beta)}{1-\beta} D^{+} g_{n}(0) \int_{\Omega} \phi_{u_{n}} u_{n}^{2} .
\end{aligned}
$$

From (89) and (70), we have

$$
\|\varphi\| \geq \frac{\|\varphi\|}{n} \geq \frac{D^{+} g_{n}(0)}{1-\beta}\left[(1+\beta) a\left\|u_{n}\right\|^{2}\right.
$$

$$
\begin{aligned}
& +(3+\beta) b\left\|u_{n}\right\|^{4}-(3+\beta) \eta \int_{\Omega} \phi_{u_{n}} u_{n}^{2} \\
& \left.-\frac{1-\beta}{n}\left\|u_{n}\right\|\right]+\frac{a(1+\beta)}{1-\beta} \int_{\Omega} \nabla u_{n} \nabla \varphi d x
\end{aligned}
$$




$$
\begin{aligned}
& +\frac{b(3+\beta)}{1-\beta}\left\|u_{n}\right\|^{2} \int_{\Omega} \nabla u_{n} \nabla \varphi-\frac{\eta(3+\beta)}{1-\beta} \\
& \cdot \int_{\Omega} \phi_{u_{n}} u_{n} \varphi \geq \frac{D^{+} g_{n}(0)}{1-\beta}\left[\frac{1-\beta}{2} C_{1}\right]+\frac{a(1+\beta)}{1-\beta} \\
& \cdot \int_{\Omega} \nabla u_{n} \nabla \varphi d x+\frac{b(3+\beta)}{1-\beta}\left\|u_{n}\right\|^{2} \int_{\Omega} \nabla u_{n} \nabla \varphi \\
& -\frac{\eta(3+\beta)}{1-\beta} \int_{\Omega} \phi_{u_{n}} u_{n} \varphi, \quad \forall n \geq N .
\end{aligned}
$$

From (85) and (90), it is easy to see that (87) holds. Thus, (86) and (87) imply that

$$
\left|D^{+} g_{n}(0)\right| \leq C, \quad \forall n \geq N,
$$

where $C>0$ is independent of $n$.

From condition (ii) and (13), we can obtain that

$$
\begin{aligned}
& \frac{1}{n}\left[\left|g_{n}(s)-1\right| \cdot\left\|u_{n}\right\|+s g_{n}(s)\|\varphi\|\right] \\
& \geq \frac{1}{n}\left\|g_{n}(s)\left(u_{n}+s \varphi\right)-u_{n}\right\| \\
& \geq J_{\lambda}\left(u_{n}\right)-J_{\lambda}\left[g_{n}(s)\left(u_{n}+s \varphi\right)\right] \\
&=-\left\{\frac{g_{n}^{2}(s)-1}{2}\right\} a\left\|u_{n}\right\|^{2}-\left\{\frac{g_{n}^{4}(s)-1}{4}\right\} b\left\|u_{n}\right\|^{4} \\
&+\left\{\frac{g_{n}^{4}(s)-1}{4}\right\} \eta \int_{\Omega} \phi_{u_{n}}^{2} u_{n}^{2} \\
&+\frac{\lambda}{1-\beta}\left[g_{n}^{1-\beta}(s)-1\right] \int_{\Omega} f(x)\left|u_{n}\right|^{1-\beta} \\
&+\frac{g_{n}^{2}(s)}{2} a\left(\left\|u_{n}\right\|^{2}-\left\|u_{n}+s \varphi\right\|^{2}\right) \\
&+\frac{g_{n}^{4}(s)}{4} b\left(\left\|u_{n}\right\|^{4}-\left\|u_{n}+s \varphi\right\|^{4}\right) \\
&+\frac{g_{n}^{4}(s)}{4} \eta \int_{\Omega}\left[\phi_{u_{n}+s \varphi}\left(u_{n}+s \varphi\right)^{2}-\phi_{u_{n}} u_{n}^{2}\right] \\
&+\frac{\lambda}{1-\beta} g_{n}^{1-\beta}(s) \int_{\Omega} f(x)\left[\left(u_{n}+s \varphi\right)^{1-\beta}-u_{n}^{1-\beta}\right] .
\end{aligned}
$$

Dividing by $s>0$ and letting $s \rightarrow 0^{+}$, we derive that

$$
\begin{aligned}
& \frac{1}{n}\left(D^{+} g_{n}(0)\left\|u_{n}\right\|+\|\varphi\|\right) \\
& \geq-a \int_{\Omega} \nabla u_{n} \nabla \varphi-b\left\|u_{n}\right\|^{2} \int_{\Omega} \nabla u_{n} \nabla \varphi \\
& \quad+\eta \int_{\Omega} \phi_{u_{n}} u_{n} \varphi d x \\
& \quad+\varliminf_{s \rightarrow 0^{+}} \frac{\lambda}{1-\beta} \int_{\Omega} \frac{f(x)\left[\left(u_{n}+s \varphi\right)^{1-\beta}-u_{n}^{1-\beta}\right]}{s} d x .
\end{aligned}
$$

Since $f(x)\left[\left(u_{n}+s \varphi\right)^{1-\beta}-u_{n}^{1-\beta}\right] \geq 0, \forall x \in \Omega, t>0$, then by Fatou's Lemma we obtain

$$
\begin{aligned}
& \int_{\Omega} f(x) u_{n}^{-\beta} \varphi d x \\
& \quad \leq \lim _{s \rightarrow 0^{+}} \frac{1}{1-\beta} \int_{\Omega} \frac{f(x)\left[\left(u_{n}+s \varphi\right)^{1-\beta}-u_{n}^{1-\beta}\right]}{s} d x .
\end{aligned}
$$

Combining (93) and (94) we deduce

$$
\begin{aligned}
\lambda \int_{\Omega} f(x) u_{n}^{-\beta} \varphi d x \leq & \frac{1}{n}\left(\left|D^{+} g_{n}(0)\right|\left\|u_{n}\right\|+\|\varphi\|\right) \\
& +\left(a+b\left\|u_{n}\right\|^{2}\right) \int_{\Omega} \nabla u_{n} \nabla \varphi \\
& -\eta \int_{\Omega} \phi_{u_{n}} u_{n} \varphi d x \\
\leq & \frac{C_{0} C+\|\varphi\|}{n} \\
& +\left(a+b\left\|u_{n}\right\|^{2}\right) \int_{\Omega} \nabla u_{n} \nabla \varphi d x \\
& -\eta \int_{\Omega} \phi_{u_{n}} u_{n} \varphi d x
\end{aligned}
$$

for all $n \geq N$, which implies that

$$
\begin{aligned}
& \lambda \underset{n \rightarrow \infty}{\lim _{\Omega}} \int_{\Omega} f(x) u_{n}^{-\beta} \varphi d x \\
& \quad \leq\left(a+b \varliminf_{n \rightarrow \infty}\left\|u_{n}\right\|^{2}\right) \int_{\Omega} \nabla u_{0} \nabla \varphi-\eta \int_{\Omega} \phi_{u_{0}} u_{0} \varphi d x .
\end{aligned}
$$

Using Fatou's Lemma, we infer that

$$
\begin{aligned}
\lambda \int_{\Omega} f(x) u_{0}^{-\beta} \varphi d x \leq & \left(a+b \varliminf_{n \rightarrow \infty}\left\|u_{n}\right\|^{2}\right) \int_{\Omega} \nabla u_{0} \nabla \varphi \\
& -\eta \int_{\Omega} \phi_{u_{0}} u_{0} \varphi d x .
\end{aligned}
$$
in (97)

On one hand, since $u_{0}(x) \geq 0$ a.e. in $\Omega$, choosing $\varphi=u_{0}$

$$
\begin{aligned}
& \left(a+b \underset{n \rightarrow \infty}{\lim }\left\|u_{n}\right\|^{2}\right)\left\|u_{0}\right\|^{2} \\
& \quad \geq \eta \int_{\Omega} \phi_{u_{0}} u_{0}^{2} d x+\lambda \int_{\Omega} f(x) u_{0}^{1-\beta} d x .
\end{aligned}
$$
that

On the other hand, according to (67) and (68), it follows

$$
\begin{aligned}
& \left(a+b \underline{\lim }_{n \rightarrow \infty}\left\|u_{n}\right\|^{2}\right)\left\|u_{0}\right\|^{2} \\
& \leq\left(a+b \varlimsup_{n \rightarrow \infty}\left\|u_{n}\right\|^{2}\right) \varlimsup_{n \rightarrow \infty}\left\|u_{n}\right\|^{2} \\
& \quad=\eta \int_{\Omega} \phi_{u_{0}} u_{0}^{2} d x+\lambda \int_{\Omega} f(x) u_{0}^{1-\beta} d x .
\end{aligned}
$$


Combining (98) and (99) yields

$$
\varliminf_{n \rightarrow \infty}\left\|u_{n}\right\|^{2}=\varlimsup_{n \rightarrow \infty}\left\|u_{n}\right\|^{2}=\left\|u_{0}\right\|^{2} .
$$

Thus, according to (97) and (100), we have

$$
\begin{aligned}
(a+b & \left.\left\|u_{0}\right\|^{2}\right) \int_{\Omega} \nabla u_{0} \nabla \varphi d x-\eta \int_{\Omega} \phi_{u_{0}} u_{0} \varphi d x \\
& -\lambda \int_{\Omega} f(x) u_{0}^{-\beta} \varphi \geq 0
\end{aligned}
$$

for all $\varphi \in H_{0}^{1}(\Omega)$ with $\varphi>0$. Therefore, by the idea of approximation and (101), we get

$$
\int_{\Omega} \nabla u_{0} \nabla \varphi d x \geq 0, \quad \forall \varphi \in H_{0}^{1}(\Omega), \varphi \geq 0
$$

$u_{0} \geq 0$ and $u_{0} \neq 0$ in $\Omega$. By the strong maximum principle for weak solutions, we obtain that $u_{0}>0$ a.e. in $\Omega$.

Second, we prove that $u_{0}$ is a solution of problem (12). In fact, we can repeat the proof of Theorem 2 and obtain that

$$
\begin{aligned}
\left(a+b\left\|u_{0}\right\|^{2}\right) \int_{\Omega} \nabla u_{0} \nabla \varphi d x-\eta \int_{\Omega} \phi_{u_{0}} u_{0} \varphi d x \\
-\lambda \int_{\Omega} f(x) u_{0}^{-\beta} \varphi d x=0, \quad \forall \varphi \in H_{0}^{1}(\Omega) .
\end{aligned}
$$

Then $u_{0} \in H_{0}^{1}(\Omega)$ is a positive solution of (12) for $\varepsilon=-1,0<$ $\lambda<\lambda_{*}$, and $\eta>\eta_{0}$.

Finally, we prove that $u_{0} \in N_{\lambda}^{+}$. According to (71) and (100), we have

$$
\begin{gathered}
a(1+\beta)\left\|u_{0}\right\|^{2}+b(3+\beta)\left\|u_{0}\right\|^{4} \\
>\eta(3+\beta) \int_{\Omega} \phi_{u_{0}} u_{0}^{2} d x
\end{gathered}
$$

which implies that $u_{0} \in N_{\lambda}^{+}$.

By Lemma 8 , we know that $N_{\lambda}^{-}$is a closed set in $H_{0}^{1}(\Omega)$ for $\lambda \in\left(0, \lambda_{*}\right)$. Thus, applying Lemma 9 and Ekeland's variational principle, we can find a bounded and nonnegative sequence $\left\{v_{n}\right\} \subset N_{\lambda}^{-}$and $v_{0} \in H_{0}^{1}(\Omega)$ such that

(i) $J_{\lambda}\left(v_{n}\right) \leq \inf _{u \in N_{\lambda}^{-}} J_{\lambda}(u)+1 / n^{2}$,

(ii) $J_{\lambda}(v) \geq J_{\lambda}\left(v_{n}\right)-(1 / n)\left\|v-v_{n}\right\|, v \in N_{\lambda}^{-}$,

(iii) $v_{n} \rightarrow v_{0}$ in $H_{0}^{1}(\Omega)$,

(iv) $v_{n} \rightarrow v_{0}$ in $L^{p}(\Omega), p \in[1,6)$,

(v) $v_{n}(x) \rightarrow v_{0}(x)$, a.e. in $\Omega$.

At this point we can repeat the proof as above and conclude that $v_{0} \in N_{\lambda}^{-}$is another positive solution of (12) for $\varepsilon=$ $-1,0<\lambda<\lambda_{*}$, and $\eta>\eta_{0}$.

\section{Conflicts of Interest}

The authors declare that they have no conflicts of interest.

\section{Acknowledgments}

This research is supported by the Reward Fund for Excellent Young and Middle-Aged Scientists of Shandong Province (BS2011SF022), China.

\section{References}

[1] Q. Zhang, "Existence, uniqueness and multiplicity of positive solutions for Schrodinger-Poisson system with singularity," Journal of Mathematical Analysis and Applications, vol. 437, no. 1, pp. 160-180, 2016.

[2] A. C. Lazer and P. J. McKenna, "On a singular nonlinear elliptic boundary-value problem," Proceedings of the American Mathematical Society, vol. 111, no. 3, pp. 721-730, 1991.

[3] A. V. Lair and A. W. Shaker, "Classical and weak solutions of a singular semilinear elliptic problem," Journal of Mathematical Analysis and Applications, vol. 211, no. 2, pp. 371-385, 1997.

[4] K. Perera and Z. Zhang, "Nontrivial solutions of Kirchhoff-type problems via the Yang index," Journal of Differential Equations, vol. 221, no. 1, pp. 246-255, 2006.

[5] X. He and W. Zou, "Infinitely many positive solutions for Kirchhoff-type problems," Nonlinear Analysis: Theory, Methods \& Applications, vol. 70, no. 3, pp. 1407-1414, 2009.

[6] A. Mao and S. Luan, "Sign-changing solutions of a class of nonlocal quasilinear elliptic boundary value problems," Journal of Mathematical Analysis and Applications, vol. 383, no. 1, pp. 239-243, 2011.

[7] C.-Y. Chen, Y.-C. Kuo, and T.-F. Wu, “The Nehari manifold for a Kirchhoff type problem involving sign-changing weight functions," Journal of Differential Equations, vol. 250, no. 4, pp. 1876-1908, 2011.

[8] X. Liu and Y. Sun, "Multiple positive solutions for Kirchhoff type problems with singularity," Communications on Pure and Applied Analysis, vol. 12, no. 2, pp. 721-733, 2013.

[9] C.-Y. Lei, J.-F. Liao, and C.-L. Tang, "Multiple positive solutions for Kirchhoff type of problems with singularity and critical exponents," Journal of Mathematical Analysis and Applications, vol. 421, no. 1, pp. 521-538, 2015.

[10] F. Li, Z. Song, and Q. Zhang, "Existence and uniqueness results for Kirchhoff-Schrödinger-Poisson system with general singularity," Applicable Analysis, pp. 1-11, 2016.

[11] J.-F. Liao, P. Zhang, J. Liu, and C.-L. Tang, "Existence and multiplicity of positive solutions for a class of Kirchhoff type problems with singularity," Journal of Mathematical Analysis and Applications, vol. 430, no. 2, pp. 1124-1148, 2015. 


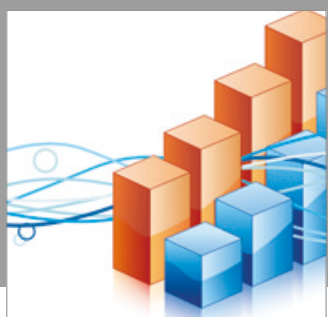

Advances in

Operations Research

vatersals

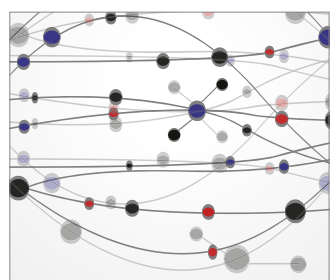

\section{The Scientific} World Journal
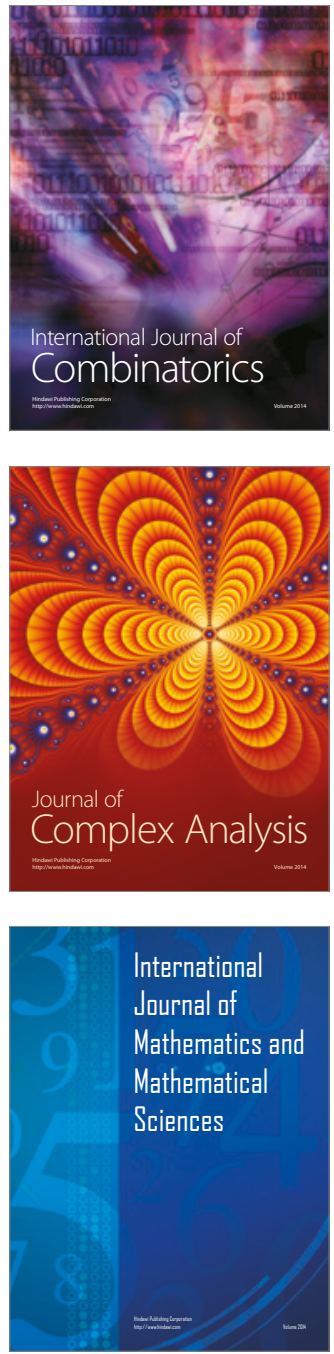
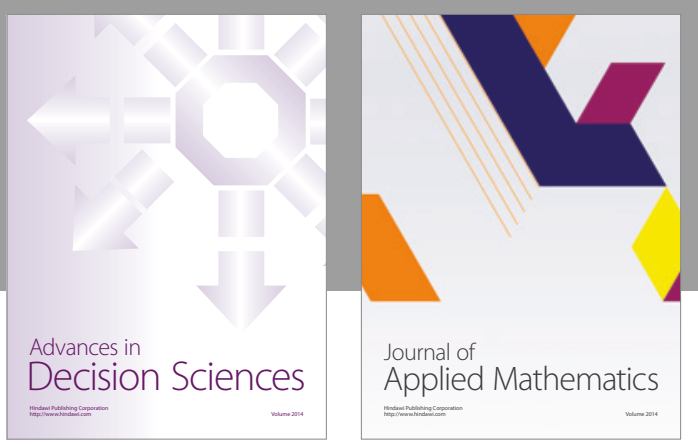

Algebra

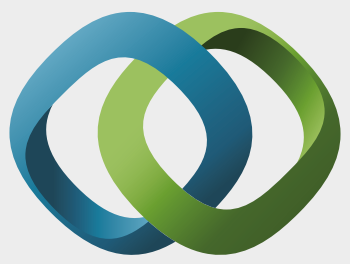

\section{Hindawi}

Submit your manuscripts at

https://www.hindawi.com
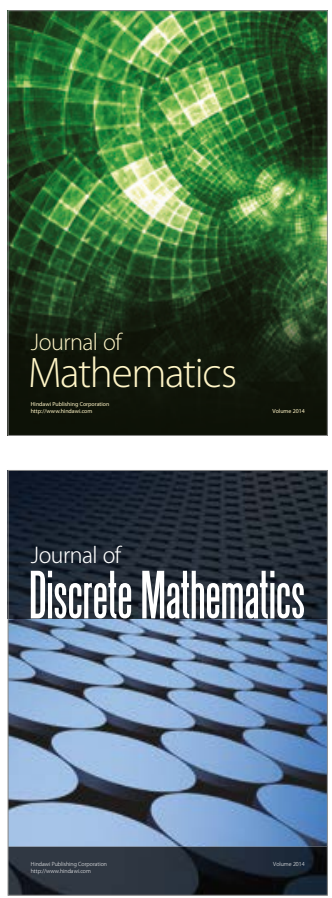

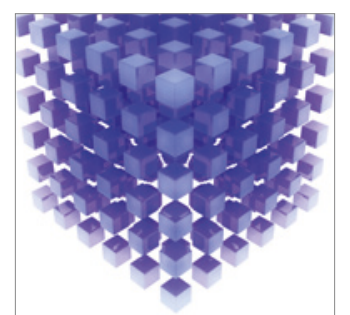

Mathematical Problems in Engineering
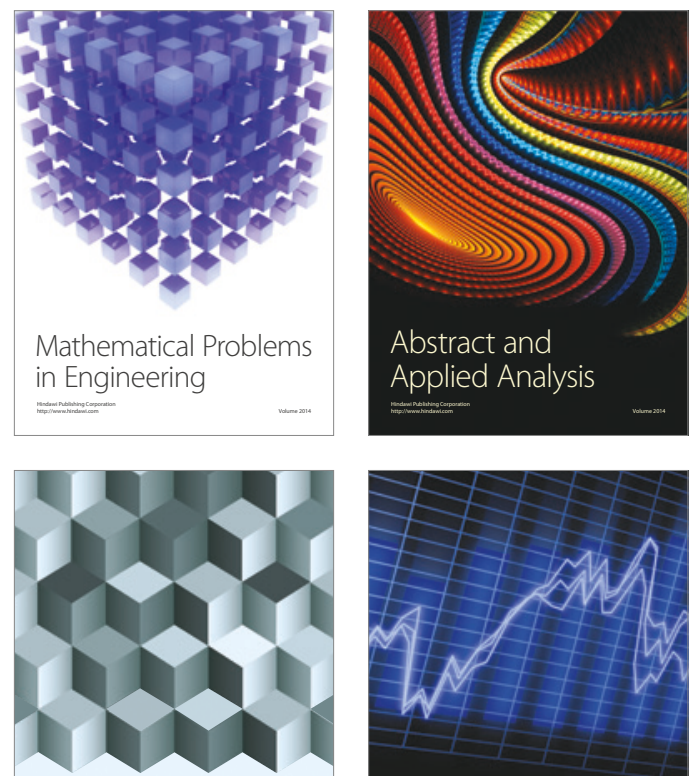

Journal of

Function Spaces

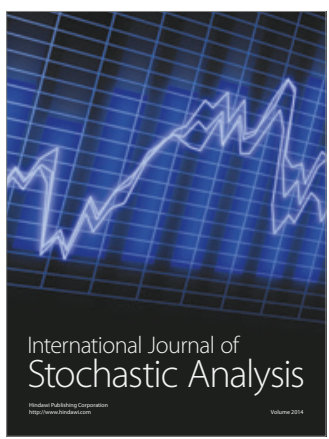

Probability and Statistics
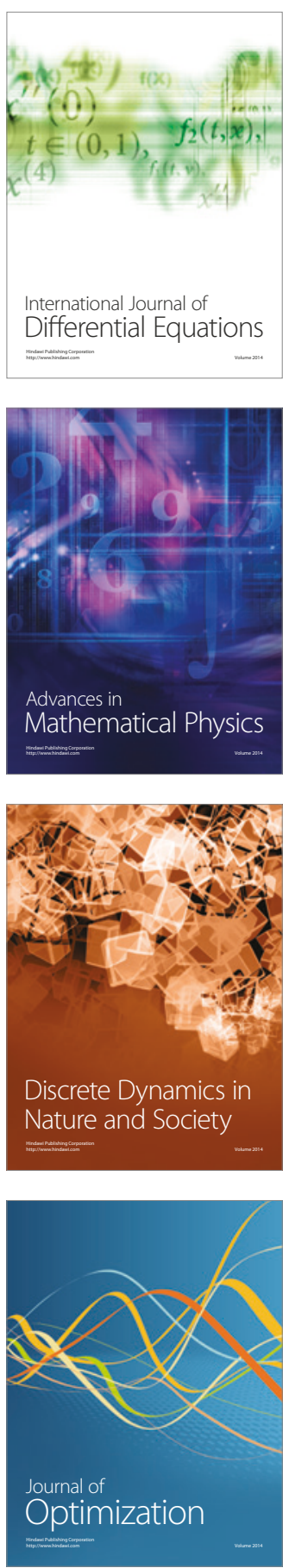